\title{
THz-Scale Field-Induced Spin Dynamics in Ferrimagnetic Iron Garnets
}

\author{
T. G. H. Blank®, ${ }^{1}$ K. A. Grishunin $\odot,{ }^{1,2}$ E. A. Mashkovich $\odot,{ }^{1,3}$ M. V. Logunov, ${ }^{4}$ A. K. Zvezdin, ${ }^{5,6}$ and A. V. Kimel ${ }^{1}$ \\ ${ }^{1}$ Radboud University, Institute for Molecules and Materials, 6525 AJ Nijmegen, The Netherlands \\ ${ }^{2}$ MIREA-Russian Technological University, 119454 Moscow, Russia \\ ${ }^{3}$ University of Nizhny Novgorod, 603022 Nizhny Novgorod, Russia \\ ${ }^{4}$ Kotel'nikov Institute of Radioengineering and Electronics, 125009 Moscow, Russia \\ ${ }^{5}$ Prokhorov General Physics Institute of the Russian Academy of Sciences, 119991 Moscow, Russia \\ ${ }^{6}$ P. N. Lebedev Physical Institute of the Russian Academy of Sciences, 119991 Moscow, Russia
}

(Received 12 February 2021; accepted 10 June 2021; published 14 July 2021)

\begin{abstract}
$\mathrm{THz}$ magnetization dynamics of antiferromagnetically coupled spins in ferrimagnetic $\mathrm{Tm}_{3} \mathrm{Fe}_{5} \mathrm{O}_{12}$ is excited by a picosecond single-cycle pulse of a magnetic field and probed with the help of the magnetooptical Faraday effect. Data analysis combined with numerical modeling shows that the dynamics corresponds to the exchange mode excited by the Zeeman interaction of the THz magnetic field with the spins. We argue that THz-pump-IR-probe experiments on ferrimagnets offer a unique tool for quantitative studies of dynamics and mechanisms to control antiferromagnetically coupled spins.
\end{abstract}

DOI: 10.1103/PhysRevLett.127.037203

All magnetically ordered materials, depending on the alignment of spins, are divided into two primary classes: ferromagnets and antiferromagnets. Ferromagnets are characterized by parallel alignment of spins that results in a net magnetic moment, while spins in antiferromagnets are aligned in a mutually antiparallel way with zero net magnetization in the unperturbed state. Antiferromagnets represent the largest but least explored class of magnets that has the potential to have a dramatic impact on spintronics and other magnetic technologies. In particular, the higher frequency $(\sim \mathrm{THz})$ of spin resonances in antiferromagnets can bring the clock speed of spintronics devices into the $\mathrm{THz}$ range [1-3].

Unfortunately, proceedings in fundamental research and the development of antiferromagnetic spintronics are considerably hindered by the lack of net magnetization in antiferromagnets, as even the discovery of antiferromagnetic order itself had to wait for the advent of neutron diffraction experiments in the late 1940s [4]. This is why approaches and mechanisms allowing efficient excitation of antiferromagnetic spins in the $\mathrm{THz}$ range became a subject of not only intense, but also challenging and intriguing research. In particular, it was recently suggested that $\mathrm{THz}$ magnetic fields can excite antiferromagnetically coupled spins with a significantly higher efficiency when accounting for the new, relativistic mechanism of field derivative torque (rFDT) [5]. This torque can reach strengths comparable to the conventional Zeeman torque [6]. However, the lack of methods for quantitative detection of spins in antiferromagnets prevents these claims from experimental verification and can even lead to mistakes in interpretation of experiments [7].
Substantial progress in understanding THz light coupling to antiferromagnetic spins can be achieved by studying ferrimagnets. Despite having antiferromagnetic order, spins pointing in opposite directions are not equivalent and therefore the net magnetization is not necessarily zero. The latter greatly simplifies experimental studies, but it does not ruin the presence of $\mathrm{THz}$ spin resonances called exchange modes. The mechanisms, efficiency, and the optimal conditions for excitation of the exchange mode in ferrimagnets with the help of ultrashort $\mathrm{THz}$ magnetic field pulses have not been analyzed yet; here, we clarify these issues. Ultimately, using simulations, we conclude that the Zeeman torque plays the dominant role in the spin excitation, while other mechanisms can essentially be neglected.

The structure (crystallographic space group Ia $\overline{3} \mathrm{~d}$ ) of rareearth iron garnets gives rise to unusual magnetic properties $[8,9]$. Three of five $\mathrm{Fe}^{3+}$ ions per formula unit $\left(\mathrm{R}_{3} \mathrm{Fe}_{5} \mathrm{O}_{12}\right)$ form a sublattice with tetrahedral symmetry and are antiferromagnetically coupled to the remaining two iron ions occupying sites of octahedral symmetry. The imbalance between these iron ions results in a net magnetic moment $\mathbf{M}_{\mathrm{Fe}}$ to which the rare-earth (RE) site magnetization $\mathbf{M}_{\mathrm{RE}}$ aligns antiparallel. The result is a three-sublattice ferrimagnet with net magnetization $\mathbf{M}=\mathbf{M}_{\mathrm{RE}}+\mathbf{M}_{\mathrm{Fe}}$. The antiferromagnetic exchange between the iron sublattices is large compared to any other interactions experienced by the $\mathrm{Fe}^{3+}$ spins, justifying the approximation of treating it as a single sublattice with magnetization $\mathbf{M}_{\mathrm{Fe}}$ [10]. The REsublattice experiences the exchange field generated by this iron magnetization, while the exchange interaction within the RE-sublattice is weak and can be ignored [8], resembling a paramagnet in the exchange field of iron. 
The rare-earth iron garnet studied in this work is a $19 \mu \mathrm{m}$ film of $\mathrm{Bi}$ - and Ga-substituted thulium iron garnet $\mathrm{Tm}_{3-\mathrm{x}} \mathrm{Bi}_{\mathrm{x}} \mathrm{Fe}_{5-\mathrm{y}} \mathrm{Ga}_{\mathrm{y}} \mathrm{O}_{12}$ with targeted composition $x=1$, $y=0.8$. The film was grown by liquid phase epitaxy on a $500 \mu \mathrm{m}$ thick (111) oriented gadolinium gallium garnet substrate. The sample was doped with $\mathrm{Bi}^{3+}$ to enhance magneto-optical effects [11-13]. Previous research on films grown in this way show that the sample is characterized by a uniaxial out-of-plane type anisotropy, as the thin-film shape anisotropy is shown to be overcome by stressinduced anisotropy from a lattice mismatch between substrate and sample [14] together with a small contribution of growth-induced anisotropy due to the site preference of bismuth ions along the growth direction $[15,16]$. Consequentially, this gives an "easy axis" along the [111] crystallographic direction. The expectations are confirmed by measurements of static magneto-optical Faraday rotation as a function of magnetic field (Fig. S1 of the Supplemental Material [17]).

In the pump-probe experiment, we use optical pulses from a Ti:Sapphire amplifier with a central wavelength of $800 \mathrm{~nm}, 4 \mathrm{~mJ}$ energy per pulse, $100 \mathrm{fs}$ pulse duration, and $1 \mathrm{kHz}$ repetition rate. These pulses were employed to generate single-cycle $\mathrm{THz}$ pulses by a titled-front optical rectification technique in a lithium niobate crystal as described in Ref. [23] and discussed in detail in Ref. [24]. The generated $\mathrm{THz}$ beam was tightly focused onto the sample [25] and spatially overlapped with a low intensity optical probe beam that was chopped out beforehand from the original beam. Varying time retardation between the $\mathrm{THz}$ pump and optical probe pulse, timeresolved measurements were obtained by mapping probe polarization changes induced by the $\mathrm{THz}$ pulse using a balanced photodetector. The strength of the $\mathrm{THz}$ electric field in free space was calibrated using the Pockels effect in a thin (110)-oriented $\mathrm{GaP}$ crystal and yields a maximum peak strength of $\left|\mathbf{E}_{\mathrm{THz}}\right| \approx 1 \mathrm{MV} / \mathrm{cm}$, implying a peak magnetic field of $0.33 \mathrm{~T}$ [18]. The $\mathrm{THz}$ pulse waveform and the corresponding Fourier spectrum are shown in Fig. S4 of the Supplemental Material [17]. Both the generated $\mathrm{THz}$ and optical probe pulses are linearly polarized. The experimental geometry is schematically depicted in Fig. 1(a). The THz magnetic field is initially along the $x$ axis, but this direction can be controlled by a set of wire-grid polarizers. Note that, using this approach, a polarization rotation of $\pi / 2$ from the initial state always reduces the $\mathrm{THz}$ magnetic field by at least one half. A static external magnetic field $\mu_{0} \mathbf{H}_{\text {ext }}$ of at most $250 \mathrm{mT}$ was applied at an angle of $\sim 10^{\circ}$ with the sample plane. From static Faraday rotation measurements (see Fig. S2 of the Supplemental Material [17]), we conclude that such maximum field strength is sufficient to saturate magnetization in the garnet film into the field direction.

Figure 1(b) shows THz-induced dynamics observed as ultrafast modulations of the probe polarization $\Delta \alpha_{F}$ as a
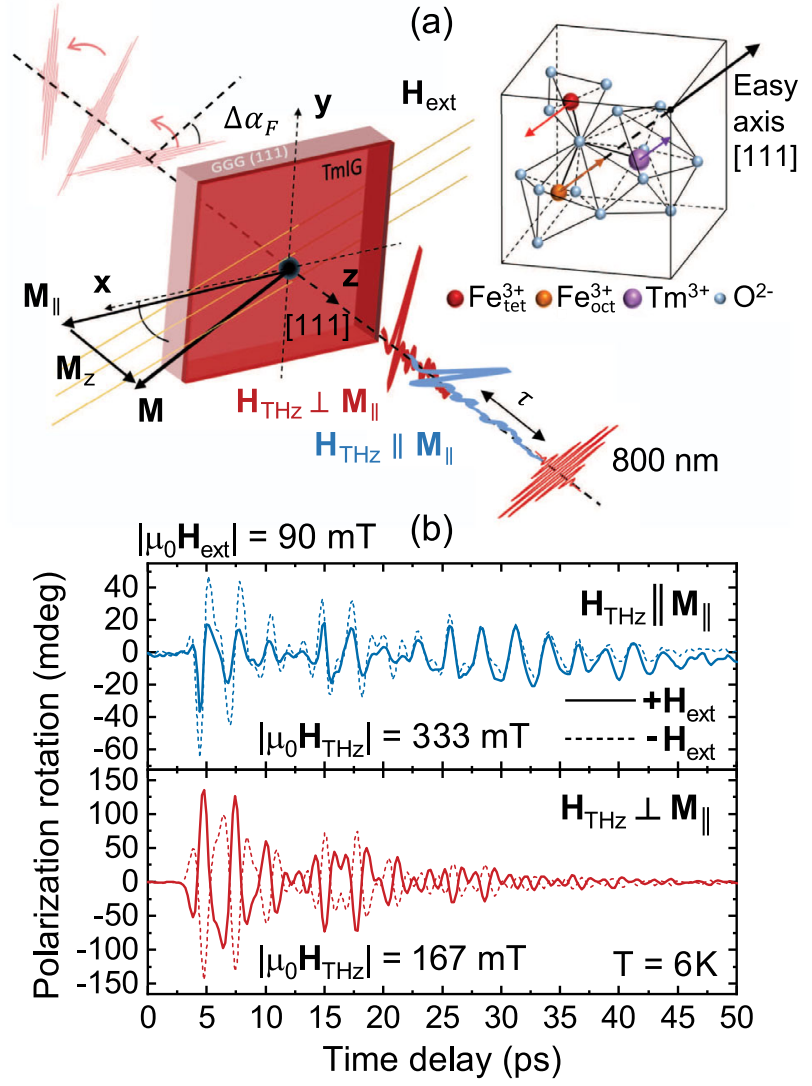

FIG. 1. (a) Schematic of the experimental setup. The illustration on the top right shows the distribution of dodecahedral $\mathrm{Tm}^{3+}$ and tetrahedral/octahedral $\mathrm{Fe}^{3+}$ ions. Any magnetic moment will tend to align along the [111] "easy axis." (b) Polarization rotation $\Delta \alpha_{F}$ measured as a function of the time delay $\tau$ between $\mathrm{THz}$ pump and optical probe pulses. Depending on the $\mathrm{THz}$ polarization, the mapped dynamics is either odd $\left(\mathbf{H}_{\mathrm{THz}} \| y \perp \mathbf{M}_{\|}\right)$with the external magnetic field or even $\left(\mathbf{H}_{\mathrm{THz}}\|x\| \mathbf{M}_{\|}\right)$. The measurements were performed at $T=6 \mathrm{~K}$.

function of the time delay $\tau$ and how it depends on the THz-pump polarization. By rotating the $\mathrm{THz}$ polarization from $\mathbf{H}_{\mathrm{THz}} \| \mathbf{M}_{\|}$to $\mathbf{H}_{\mathrm{THz}} \perp \mathbf{M}_{\|}$, the symmetry of the highfrequency oscillations with respect to the polarity of the external magnetic field is altered. To reveal the origin of these peculiar THz-induced modulations, we performed systematic studies as a function of the pump and probe polarizations, external magnetic field, THz field strength, and temperature.

The observed oscillations of the probe polarization rotation are a result of a periodic modulation of optical anisotropy (birefringence) in the sample. A THz pulse is able to induce such optical anisotropy by modifying the dielectric permittivity tensor $\epsilon_{i j}$. If one neglects dissipation, which is a safe approximation for iron garnets at the wavelength of $800 \mathrm{~nm}[13,26]$, the tensor is Hermitian [27]. This type of tensor $\epsilon_{i j}$ can be written as a sum of the symmetric (real) $\epsilon_{i j}^{(s)}=\epsilon_{j i}^{(s)}$ and antisymmetric (imaginary) 
$\epsilon_{i j}^{(a)}=-\epsilon_{j i}^{(a)}$ parts. Measurements of the THz-induced dynamics as a function of probe polarization angle show no dependency (Fig. S5 of the Supplemental Material [17]), indicating that the THz-induced modulations originate from the antisymmetric part of the dielectric tensor. It means that the polarization rotation $\Delta \alpha_{F}$ must be assigned to the magneto-optical Faraday effect. In a [111] garnet crystal, this effect is a measure of the magnetization along the $z$ axis $[28,29]$ :

$$
\epsilon_{x y}^{(a)} \sim M_{z} .
$$

When $\mathbf{H}_{\mathrm{THz}} \perp \mathbf{M}_{\|}$, changing the external magnetic field polarity from $+\mathbf{H}_{\text {ext }}$ to $-\mathbf{H}_{\text {ext }}$ flips the sign of the observed dynamics [Fig. 1(b), red waveforms]. Moreover, by increasing the strength of the static magnetic field, we found that the amplitude of the oscillations and the net magnetization saturate at the same field (Fig. S6 of the Supplemental Material [17]). This fact confirms that the THz-induced dynamics must by assigned to the dynamics of the magnetization M. And according to Eq. (1), the measurements are only sensitive to modulations of the outof-plane magnetization component. Thus, if we compare the size of the amplitude of the oscillations $\Delta \alpha_{F}$ with the saturated static Faraday rotation $\alpha_{F}\left(\sim 20^{\circ}\right.$ at $\left.800 \mathrm{~nm}\right)$, this allows us to quantitatively estimate the relative change of the magnetization along the $z$ axis during the oscillations $\Delta M_{z} / M_{z} \sim 0.012$. For another case $\mathbf{H}_{\mathrm{THz}} \| \mathbf{M}_{\|}$, the signal also saturates in line with the magnetization $\mathbf{M}$, but the phase of the oscillations is unaffected by the polarity of the external field [see Fig. 1(b)].

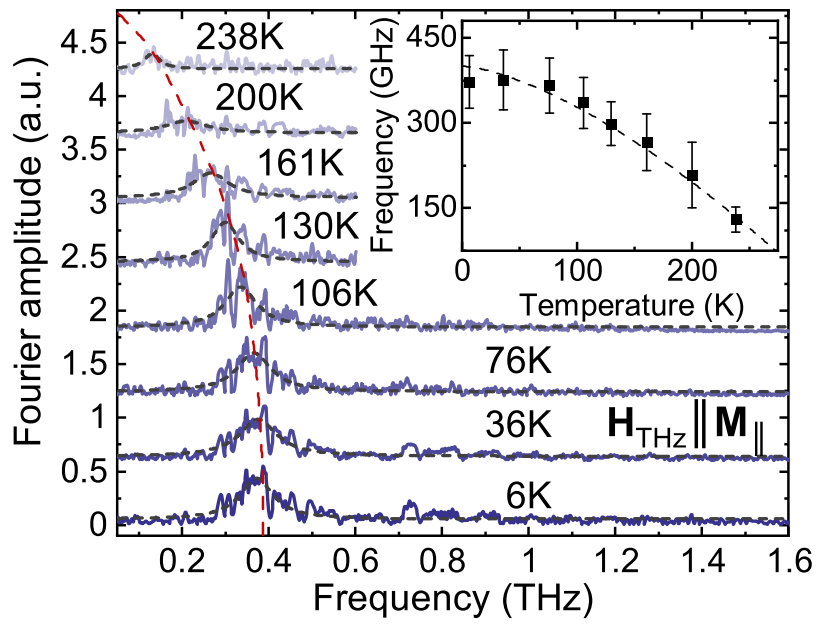

FIG. 2. Fourier spectrum of the THz-induced signal $\left(\mathbf{H}_{\mathrm{THz}} \| \mathbf{M}_{\|}\right)$measured at various temperatures. Central frequencies of the peaks deduced from the fit are plotted as a function of temperature in the inset. The dotted line denotes a fit with Eq. (8) $\left(\omega_{0} / 2 \pi=400 \mathrm{GHz}, T_{C}=314 \mathrm{~K}\right)$ and the bars denote \pm halfwidth-half-maximum of the fitted Lorentzians. The fast Fourier transform spectrum for $\mathbf{H}_{\mathrm{THz}} \perp \mathbf{M}$ is added to the Supplemental Material (Fig. S8) [17].
Figure 2 shows the Fourier spectra of the THz-induced waveforms ranging in the entire accessible temperature range when $\mathbf{H}_{\mathrm{THz}} \| \mathbf{M}_{\|}$. The inset summarizes the temperature dependence of the peak frequency, and this behavior is in qualitative agreement with what could be expected for an exchange mode in rare-earth iron garnets $[19,30]$. In order to get a better insight into the THz-induced magnetization dynamics, we modeled the response of $\mathbf{M}$ to a $\mathrm{THz}$ magnetic field pulse. Assuming the $\mathrm{THz}$ pulse does not lead to substantial heating of the medium, the longitudinal magnetization dynamics can be neglected and the ferrimagnet can be modeled as two macrospins obeying the Landau-Lifshitz-Gilbert equations [20,31]. Far from the compensation point and the Curie temperature, such a model is expected to provide an adequate description of spins dynamics in ferrimagnets [32,33]. The equations, in particular, account for the rFDT derived in Ref. [5]:

$\frac{d \mathbf{M}_{i}}{d t}=-\gamma_{i} \mathbf{M}_{i} \times \mathbf{B}_{i}^{\mathrm{eff}}(t)+\frac{\alpha_{i}}{M_{i}} \mathbf{M}_{i} \times\left(\frac{d \mathbf{M}_{i}}{d t}+\frac{a_{i}^{3}}{\mu_{B}} \frac{d \mathbf{H}}{d t}\right)$,

where we take $\mathbf{M}_{i}$ with $i=\mathrm{Fe}, \mathrm{Tm}$ to be nominal magnetizations of the macroscopic iron and thulium sublattices defined as the amount of $\mu_{B}$ per formula unit $\mathrm{R}_{3} \mathrm{Fe}_{5} \mathrm{O}_{12}$. We use literature $g$ values for thulium $g_{\mathrm{Tm}}=$ $7 / 6$ and iron $g_{\mathrm{Fe}}=2$ [34]. Based on the Ga content, we assume for the net iron sublattice magnetization $\left|\mathbf{M}_{\mathrm{Fe}}\right|=$ $4.2\left(\mu_{B}\right.$ per formula unit $\left.\mathrm{R}_{3} \mathrm{Fe}_{5} \mathrm{O}_{12}\right)$ [34], antiferromagnetically coupled to the magnetization of thulium $\left|\mathbf{M}_{\mathrm{Tm}}\right|=2$. The latter is taken to match the effective $g$ factor $g_{\mathrm{ef}} \equiv\left(M_{\mathrm{Fe}}-M_{\mathrm{Tm}}\right) /\left(\left(M_{\mathrm{Fe}} / g_{\mathrm{Fe}}\right)-M_{\mathrm{Tm}} / g_{\mathrm{Tm}}\right) \approx 6$ determined from the slope of the ferromagnetic resonance frequency versus external magnetic field (Fig. S10 of the Supplemental Material [17]). The volume of the unit cell $a_{i}^{3}$ [35] per spin constitutes a small factor $a^{3} / \mu_{B} \sim 10^{-5} \mathrm{~m} / \mathrm{A}$. The effective magnetic fields $\mathbf{B}_{i}^{\text {eff }} \equiv-\delta \Phi / \delta \mathbf{M}_{i}$ (in T) are derived from the thermodynamic potential $\Phi$ [36], containing exchange interaction and Zeeman coupling to the external field and $\mathrm{THz}$ magnetic field $\mathbf{H}(t)$ (in $\mathrm{A} / \mathrm{m}$ ). For the model we use an estimate of the exchange constant $\Lambda=-30 \mathrm{~T} / \mu_{B}[34,37,38]$ and $\mathrm{THz}$ magnetic field modeled by the Gaussian derivative function fitted to the experimental waveform (see Fig. S11 of the Supplemental Material [17]). The initial state of the net magnetization vector is taken along the external field, considering we saturated the magnetization experimentally. The numerical solution of these equations reveals that the $\mathrm{THz}$ magnetic field induces dynamics of the Néel vector $\mathbf{L} \equiv \mathbf{M}_{\mathrm{Fe}}-\mathbf{M}_{\mathrm{Tm}}$ and the magnetization $\mathbf{M} \equiv \mathbf{M}_{\mathrm{Fe}}+\mathbf{M}_{\mathrm{Tm}}$. The dynamics of the iron magnetization $\mathbf{M}_{\mathrm{Fe}}$, which dominates the detected magneto-optical signal, is shown in Fig. 3. The phenomenological Gilbert damping factors of $\alpha_{\mathrm{Fe}}=\alpha_{\mathrm{Tm}}=0.005$ have been taken to match the 


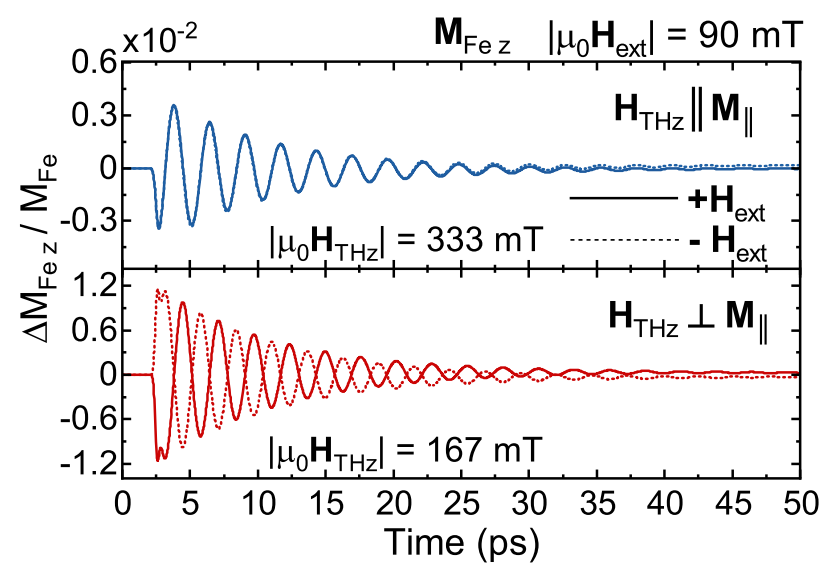

FIG. 3. Dynamics of the $z$ component of iron $\mathbf{M}_{\mathrm{Fe}}$ modeled by Landau-Lifshitz-Gilbert equations. Note that at high $\mathrm{THz}$ fluences and low temperatures, the blue curves for two field polarities are not exactly symmetric, as can be seen in Fig. 1 (b). A possible reason for this behavior could be an onset of higher-order effects in the detection of spins such as the magnetooptical Cotton-Mouton effect.

experimental observations. The simulation contains a highfrequency magnetic resonance at around $380 \mathrm{GHz}$, which we identify as the Kaplan-Kittel exchange mode since its frequency depends linearly on the exchange constant [20]. The dynamics of $M_{\mathrm{Fe}, z(t)}$ in Fig. 3 is in agreement with our experimental results in Fig. 1(b). It has a large amplitude and changes sign upon reversing $\mathbf{M}$ when $\mathbf{H}_{\mathrm{THz}} \perp \mathbf{M}_{\|}$, while the amplitude is approximately 4 times smaller and the sign is conserved if $\mathbf{H}_{\mathrm{THz}} \| \mathbf{M}_{\|}$. Besides reproducing the correct ratio of the amplitudes for the two $\mathrm{THz}$ polarizations, the estimated absolute amplitude of $\Delta M_{z} / M_{z} \sim$ 0.012 for $\mathbf{H}_{\mathrm{THz}} \perp \mathbf{M}_{\|}$also matches very well to the simulations even if the rFDT term is not taken into account. As proposed in Ref. [6], the contribution of this term will be indeed small in cases of low damping $\alpha<0.01$. However, we expect that the rFDT can play a role in systems where the magnetic sublattices have equivalent gyromagnetic ratios and the damping is large. Altogether, the simulations point out that the observed oscillations correspond to the exchange mode of spin resonance and show that Zeeman torque plays the dominant role in the excitation of this mode with $\mathrm{THz}$ magnetic field.

These conclusions could also be confirmed analytically using Lagrangian mechanics and the effective Lagrangian (see the Supplemental Material [17] for the full derivation):

$$
\begin{aligned}
\mathcal{L}_{\text {eff }}= & \frac{\mathcal{M}^{2}}{2 \delta}\left\{\left[\left(\frac{\dot{\phi}}{\bar{\gamma}}-H\right) \sin \theta+h_{y} \cos \theta \cos \phi\right]^{2}\right. \\
& \left.+\left(\frac{\dot{\theta}}{\bar{\gamma}}+h_{y} \sin \phi\right)^{2}\right\}+m\left(H-\frac{\dot{\phi}}{\gamma_{\text {ef }}}\right) \cos \theta \\
& +m h_{y} \sin \theta \cos \phi+K_{U} \sin ^{2} \theta \sin ^{2} \phi,
\end{aligned}
$$

where $\mathcal{M}=M_{\mathrm{Fe}}+M_{\mathrm{Tm}}, m=M_{\mathrm{Fe}}-M_{\mathrm{Tm}}, \delta \equiv-4 \Lambda M_{\mathrm{Fe}} M_{\mathrm{Tm}}$, $1 / \bar{\gamma}=\left(M_{\mathrm{Fe}} / \gamma_{\mathrm{Fe}}+M_{\mathrm{Tm}} / \gamma_{\mathrm{Tm}}\right) /\left(M_{\mathrm{Fe}}+M_{\mathrm{Tm}}\right), \gamma_{\mathrm{ef}}=g_{\mathrm{ef}} \mu_{B} / \hbar$, $h_{x}(t)$, and $h_{y}(t)$ are the THz magnetic field components in the sample $x-y$ plane as in Fig. 1 and $H(t) \equiv H_{\text {ext }}+h_{x}(t)$ is the total field along the external field $x$ direction (the $10^{\circ}$ inclination angle of external magnetic field is here ignored). The polar angle $\theta \in[0, \pi]$ is defined with respect to the external field $x$ axis. In this coordinate system, the net magnetization vector can be expressed as $\mathbf{M}=m(\cos \theta$, $\sin \theta \cos \phi, \sin \theta \sin \phi)$. Equations of motion now follow from the Euler-Lagrange equations, taking into account a phenomenological damping term through a Rayleigh function [21]. The results can be linearized around the groundstate angles $\theta_{0}, \phi_{0}$ found by minimization of the thermodynamic potential $\Phi$, for which we find $\phi_{0}=\pi / 2$ and $\theta_{0}$ depending on the ratio of external field to anisotropy. This has been done for general $\theta_{0}$ in the Supplemental Material [17], yielding complex equations of motion. In the special case of a zero external field, the spins lie along the easy axis of anisotropy $\left(\theta_{0}=\pi / 2\right)$. Linearizing around the groundstate angles $\theta=\theta_{0}+\theta_{l}, \phi=\phi_{0}+\phi_{l}$ with $\theta_{l}, \phi_{l} \ll 1$, the equations of motion then take the simple form

$\ddot{\theta}_{l}+\frac{\alpha \mathcal{M} \bar{\gamma}}{\chi_{\perp}} \dot{\theta}_{l}+\frac{2 K_{U} \bar{\gamma}^{2}}{\chi_{\perp}} \theta_{l}-\frac{m \bar{\gamma}^{2}}{\gamma_{\mathrm{ef}} \chi_{\perp}} \dot{\phi}_{l}=-\bar{\gamma} \dot{h}_{y}-\frac{m \bar{\gamma}^{2} h_{x}}{\chi_{\perp}}$,

$\ddot{\phi}_{l}+\frac{\alpha \mathcal{M} \bar{\gamma}}{\chi_{\perp}} \dot{\phi}_{l}+\frac{2 K_{U} \bar{\gamma}^{2}}{\chi_{\perp}} \phi_{l}+\frac{m \bar{\gamma}^{2}}{\gamma_{\mathrm{ef}} \chi_{\perp}} \dot{\theta}_{l}=\bar{\gamma} \dot{h}_{x}-\frac{m \bar{\gamma}^{2} h_{y}}{\chi_{\perp}}$.

Here, $\chi_{\perp} \equiv \mathcal{M}^{2} / \delta$ is a constant inversely proportional to the exchange constant and $\alpha$ is the Gilbert damping parameter. It can be seen that the large THz field derivative term $\gamma \dot{h}_{i}$ appears as the dominant driving force in accordance with our understanding of how dynamical $\mathrm{THz}$ fields may excite antiferromagnetic magnons in antiferromagnets (where $m \rightarrow 0$ ) by Zeeman interaction [18,39]. Moreover, each equation of motion contains a mutually orthogonal component of the field derivative $\dot{h}_{x, y}$. Noting that $\mathbf{H}_{\mathrm{THz}} \perp \mathbf{M}_{\|}$leads to $\dot{h}_{x}=0$ and $\mathbf{H}_{\mathrm{THz}} \| \mathbf{M}_{\|}$to $\dot{h}_{y}=0$, the symmetry with respect to the external field $\pm \mathbf{H}_{\text {ext }}$, as observed experimentally, can now be explained (see the Supplemental Material [17]).

Moreover, considering free precession $\alpha \rightarrow 0, h_{x, y} \rightarrow 0$, the absolute eigenfrequencies of the coupled set of equations [Eqs. (4) and (5)] are

$$
\begin{aligned}
\omega_{\mathrm{ex}}=\frac{m \bar{\gamma}^{2}}{\gamma_{\mathrm{ef}} \chi_{\perp}} \approx|\Lambda|\left(\left|\gamma_{\mathrm{Tm}}\right| M_{\mathrm{Fe}}-\left|\gamma_{\mathrm{Fe}}\right| M_{\mathrm{Tm}}\right), \\
\omega_{\mathrm{FM}}=\gamma_{\mathrm{ef}} \frac{2 K_{U}}{m} \equiv \gamma_{\mathrm{ef}} H_{a} .
\end{aligned}
$$


Equation (6) corresponds to Kaplan-Kittel's exchange resonance frequency [20], while Eq. (7) describes the conventional ferromagnetic precession of the net magnetization in the anisotropy field $H_{a}$. Using Eq. (6) and Bloch's law for the spontaneous magnetization of iron while $M_{\mathrm{Tm}}(T) \sim M_{\mathrm{Fe}}(T)$, we fitted the temperature dependence of the frequency shown in the inset of Fig. 2 using

$$
\omega_{\mathrm{ex}}(T) \sim \omega_{0}\left[1-\left(T / T_{C}\right)^{\frac{3}{2}}\right],
$$

where $\omega_{0}$ is the exchange resonance frequency at zero Kelvin and $T_{C}$ the Curie temperature. In reality, $M_{\mathrm{Tm}}$ drops faster with temperature than the magnetization of iron, accounting for the slight rise of frequency at low temperatures. In general, the fit is another confirmation of the validity of our assumption that the observed oscillations correspond to the exchange mode.

In conclusion, investigating the response of ferrimagnets to $\mathrm{THz}$ fields and comparing the data to theoretical predictions from numerical solutions of the LandauLifshitz-Gilbert equations and analytical solutions derived from Euler-Lagrange equations of motion, we showed that the $\mathrm{THz}$ field excites the exchange mode in the ensemble of antiferromagnetically coupled spins. We demonstrated that the Zeeman torque plays a dominant role in the coupling of the $\mathrm{THz}$ field to the spins. While quantitative studies of spin dynamics in compensated antiferromagnets seem to require complex magnetometry techniques, ferrimagnets facilitate an excellent playground to study the dynamics of antiferromagnetically coupled spins. Finally, we would like to point out that previous measurements of ferrimagnetic resonance $[30,40]$ could only reveal an effective gyromagnetic ratio. Using an excitation of exchange mode with a THz magnetic field, magneto-optical detection via the Faraday effect, and a comparison of the relative and absolute amplitudes of the magnetization dynamics between experiment and simulation when $\mathbf{H}_{\mathrm{THz}}$ is parallel and perpendicular to $\mathbf{M}_{\|}$provides a universal technique to directly estimate the individual gyromagnetic ratio of the ions.

The authors thank S. Semin, Ch. Berkhout, and P. Albers for technical support. The work was supported by de Nederlandse Organisatie voor Wetenschappelijk Onderzoek (NWO). K. G. acknowledges the support by Russian Foundation for Basic Research Project No. 18-0240027. E. A. M. acknowledges the support by the Ministry of Science and Higher Education of the Russian Federation (075-15-2020-927). M. V. L. acknowledges the support from the Russian Foundation for Basic Research (No. 18-29-27020 and No. 18-52-16006).

[1] V. Baltz, A. Manchon, M. Tsoi, T. Moriyama, T. Ono, and Y. Tserkovnyak, Antiferromagnetic spintronics, Rev. Mod. Phys. 90, 015005 (2018).
[2] P. Němec, M. Fiebig, T. Kampfrath, and A. V. Kimel, Antiferromagnetic opto-spintronics, Nat. Phys. 14, 229 (2018).

[3] T. Jungwirth, X. Marti, P. Wadley, and J. Wunderlich, Antiferromagnetic spintronics, Nat. Nanotechnol. 11, 231 (2016).

[4] C. G. Shull and J. S. Smart, Detection of antiferromagnetism by neutron diffraction, Phys. Rev. 76, 1256 (1949).

[5] R. Mondal, M. Berritta, and P. M. Oppeneer, Relativistic theory of spin relaxation mechanisms in the LandauLifshitz-Gilbert equation of spin dynamics, Phys. Rev. B 94, 144419 (2016).

[6] R. Mondal, A. Donges, U. Ritzmann, P. M. Oppeneer, and U. Nowak, Terahertz spin dynamics driven by a fieldderivative torque, Phys. Rev. B 100, 060409(R) (2019).

[7] N. Bhattacharjee, A. A. Sapozhnik, S. Y. Bodnar, V. Y. Grigorev, S. Y. Agustsson, J. Cao, D. Dominko, M. Obergfell, O. Gomonay, J. Sinova, M. Kläui, H.-J. Elmers, M. Jourdan, and J. Demsar, Retraction: Néel Spin-Orbit Torque Driven Antiferromagnetic Resonance in $\mathrm{Mn}_{2} \mathrm{Au}$ Probed by TimeDomain THz Spectroscopy, Phys. Rev. Lett. 120, 237201 (2018); Erratum, Phys. Rev. Lett. 124, 039901 (2020).

[8] A. E. Clark and E. Callen, Néel ferrimagnets in large magnetic fields, J. Appl. Phys. 39, 5972 (1968).

[9] A. M. Kalashnikova, V. V. Pavlov, A. V. Kimel, A. Kirilyuk, Th. Rasing, and R. V. Pisarev, Magneto-optical study of holmium iron garnet $\mathrm{Ho}_{3} \mathrm{Fe}_{5} \mathrm{O}_{12}$, Low Temp. Phys. 38, 863 (2012).

[10] R. Z. Levitin, B. K. Ponomarev, and Yu. F. Popov, Magnetization of iron garnets of heavy rare earth elements in fields up to $240 \mathrm{kOe}$, Sov. Phys. JETP 32, 1056 (1971), http://jetp.ac.ru/cgi-bin/dn/e_032_06_1056.pdf.

[11] P. Hansen and W. Tolksdorf, Magnetic and magneto-optic properties of bismuth-substituted thulium iron-garnet films, J. Appl. Phys. 69, 4577 (1991).

[12] T. Hibiya, Y. Morishige, and J. Nakashima, Growth and characterization of liquid-phase epitaxial Bi-substituted iron garnet films for magneto-optic application, Jpn. J. Appl. Phys. 24, 1316 (1985).

[13] A. K. Zvezdin and V. A. Kotov, Modern Magnetooptics and Magnetooptical Materials, Condensed Matter Physics (CRC Press, Boca Raton, 1997).

[14] M. Kubota, A. Tsukazaki, F. Kagawa, K. Shibuya, Y. Tokunaga, M. Kawasaki, and Y. Tokura, Stress-induced perpendicular magnetization in epitaxial iron garnet thin films, Appl. Phys. Express 5, 103002 (2012).

[15] R. Gerhardt, S. Sure, H. Dtsch, T. Linkewitz, and W. Tolksdorf, Optical properties of bismuth and gallium substituted thulium iron garnet films, Opt. Commun. 102, 31 (1993).

[16] P. Hansen, C. Klages, and K. Witter, Growth-induced anisotropy and Faraday rotation of bismuth-substituted europium-iron-garnet films, J. Appl. Phys. 63, 2058 (1988).

[17] See Supplemental Material, which includes Refs. [9,18-22], at http://link.aps.org/supplemental/10.1103/PhysRevLett .127 .037203 for magneto-optical characterization of the sample, experimental details of $\mathrm{THz}$ generation, pump and probe polarization dependencies, the amplitude of dynamics vs THz and external magnetic fields, supplemental waveforms and Fourier spectra over a wide temperature 
range, details on the numerical modeling, and a comprehensive description of the Lagrangian formalism.

[18] E. A. Mashkovich, K. A. Grishunin, R. V. Mikhaylovskiy, A. K. Zvezdin, R. V. Pisarev, M. B. Strugatsky, P. C. M. Christianen, T. Rasing, and A. V. Kimel, Terahertz Optomagnetism: Nonlinear $\mathrm{THz}$ Excitation of $\mathrm{GHz}$ Spin Waves in Antiferromagnetic $\mathrm{FeBO}_{3}$, Phys. Rev. Lett. 123, 157202 (2019).

[19] A. J. Sievers and M. Tinkham, Far infrared spectra of rareearth iron garnets, Phys. Rev. 129, 1995 (1963).

[20] J. Kaplan and C. Kittel, Exchange frequency electron spin resonance in ferrites, J. Chem. Phys. 21, 760 (1953).

[21] M. D. Davydova, K. A. Zvezdin, A. V. Kimel, and A. K. Zvezdin, Ultrafast spin dynamics in ferrimagnets with compensation point, J. Phys. Condens. Matter 32, 01LT01 (2020).

[22] M. Sajadi, M. Wolf, and T. Kampfrath, Terahertz-fieldinduced optical birefringence in common window and substrate materials, Opt. Express 23, 28985 (2015).

[23] J. Hebling, G. Almási, I. Z. Kozma, and J. Kuhl, Velocity matching by pulse front tilting for large-area THz-pulse generation, Opt. Express 10, 1161 (2002).

[24] H. Hirori, A. Doi, F. Blanchard, and K. Tanaka, Single-cycle terahertz pulses with amplitudes exceeding $1 \mathrm{MV} / \mathrm{cm}$ generated by optical rectification in $\mathrm{LiNbO}_{3}, \mathrm{Appl}$. Phys. Lett. 98, 091106 (2011).

[25] H. Hirori and K. Tanaka, Dynamical nonlinear interactions of solids with strong terahertz pulses, J. Phys. Soc. Jpn. 85, 082001 (2016).

[26] D. L. Wood and J. P. Remeika, Effect of impurities on the optical properties of yttrium iron garnet, J. Appl. Phys. 38, 1038 (1967).

[27] L. D. Landau and E. M. Lifshitz, Electrodynamics of Continious Media, Course of Theoretical Physics Vol. 8 (Pergamon Press, New York, 1960).

[28] R. R. Birss, Symmetry and Magnetism, Selected Topics in Solid State Physics (North-Holland Pub. Co., Amsterdam, 1964).

[29] R. V. Pisarev, B. B. Krichevtsov, V. N. Gridnev, V. P. Klin, D. Frohlich, and C. Pahlke-Lerch, Optical second-harmonic generation in magnetic garnet thin films, J. Phys. Condens. Matter 5, 8621 (1993).

[30] A. H. M. Reid, A. V. Kimel, A. Kirilyuk, J. F. Gregg, and T. Rasing, Optical Excitation of a Forbidden Magnetic Resonance Mode in a Doped Lutetium-Iron-Garnet Film via the Inverse Faraday Effect, Phys. Rev. Lett. 105, 107402 (2010).

[31] A. G. Gurevich and G. A. Melkov, Magnetization Oscillations and Waves (Taylor \& Francis, London, 1996).

[32] U. Atxitia, D. Hinzke, and U. Nowak, Fundamentals and applications of the Landau-Lifshitz-Bloch equation, J. Phys. D 50, 033003 (2017).

[33] F. Schlickeiser, U. Atxitia, S. Wienholdt, D. Hinzke, O. Chubykalo-Fesenko, and U. Nowak, Temperature dependence of the frequencies and effective damping parameters of ferrimagnetic resonance, Phys. Rev. B 86, 214416 (2012).

[34] E. P. Wohlfarth, Handbook of Magnetic Materials, Ferromagnetic Materials: A Handbook on the Properties of Magnetically Ordered Substances Vol. 2 (Elsevier Science, New York, 1986).

[35] We have used the following set of values for calculating the magnitude of rFDT terms: $a_{\mathrm{Fe}}^{3}=1.221 \times 10^{-28} \mathrm{~m}^{3}$, $a_{\mathrm{Tm}}^{3}=5.815 \times 10^{-29} \mathrm{~m}^{3}$, and vacuum permeability $\mu_{0}=1.257 \times 10^{6} \mathrm{Tm} \mathrm{A}^{-1}$.

[36] A. Kirilyuk, A. V. Kimel, and T. Rasing, Ultrafast optical manipulation of magnetic order, Rev. Mod. Phys. 82, 2731 (2010).

[37] G. F. Dionne and P. F. Tumelty, Molecular-field coefficients of $\mathrm{Tm}_{3} \mathrm{Fe}_{5} \mathrm{O}_{12}$, J. Appl. Phys. 50, 8257 (1979).

[38] G. F. Dionne, Molecular field and exchange constants of $\mathrm{Gd}^{3+}$-substituted ferrimagnetic garnets, J. Appl. Phys. 42, 2142 (1971).

[39] A. V. Kimel, A. M. Kalashnikova, A. Pogrebna, and A. K. Zvezdin, Fundamentals and perspectives of ultrafast photoferroic recording, Phys. Rep. 852, 1 (2020).

[40] J. Becker, A. Tsukamoto, A. Kirilyuk, J. C. Maan, T. Rasing, P. C. M. Christianen, and A. V. Kimel, Ultrafast Magnetism of a Ferrimagnet across the Spin-Flop Transition in High Magnetic Fields, Phys. Rev. Lett. 118, 117203 (2017). 